\title{
Factors Contributing to HAZMAT-Related Accidents in Marine Transportation
}

\author{
Mohd. N. Yacob ${ }^{1}$, Mimi H.Hassim*2 \\ ${ }^{1}$ Faculty of Engineering Technology, Universiti Malaysia Pahang, Lebuhraya Tun Razak, 26300 Kuantan, \\ Pahang, Malaysia \\ ${ }^{2}$ Faculty of Chemical and Energy Engineering, UniversitiTeknologi Malaysia, 81310 Johor Bahru, Johor, \\ Malaysia \\ *Corresponding author: mimi@cheme.utm.my
}

\author{
Article History \\ Received: Jan 10, 2018 \\ Received in revised form: July 07, 2018 \\ Accepted: July 09, 2018 \\ Published: July 30, 2018
}

\begin{abstract}
The transportation of hazardous materials (HAZMAT) especially via sea has continuously become an international concern with the continual increase in the number of accidents reported by the industries in recent years. The accidents have resulted in various effects ranging from insignificant damages to very severe losses particularly on the environment and property. In order to avoid such accidents or reduce the risks of having one, it is vital to primarily understand the factors that could lead to marine transportation accidents involving HAZMAT. Surveys were conducted among 24 representatives from various shipping industries in Malaysia. The purpose was for the representatives to assign the appropriate ranking point for each factor based on their experiences and opinions on the significance of the factors in causing accidents. This information is valuable in serving as an initial screening for safety assessment in HAZMAT marine transport operation so that the potential hazards can be avoided or reduced. Based on the assessment results using the factors selected from the surveys, a more comprehensive assessment that is particularly relevant to enhance the existing safety assessment in HAZMAT operation can then be performed towards achieving an inherently safer HAZMAT marine transportation.
\end{abstract}

Keywords: HAZMAT, marine transportation accident, marine transport operation, risk, hazard, safety assessment.

\subsection{INTRODUCTION}

The risks and hazards of marine transport operation of hazardous materials (HAZMAT) are widely known and have drawn the attention of many in the marine transportation industries worldwide. Despite being recognized as a critical issue for marine transportation industries, marine transportation still plays a major role in the rapid development of the modern world economy. Generally, the growth of marine transportation industries is attributed by the high performance of comprehensive logistic or international trade over the years. The continuous demand of marine transportation is fuelled by the need to accommodate growing ship operations, especially for HAZMAT transportation. In fact, the number of demands fortransporting HAZMAT has continuously increased, which is more than tripled since 1970 [1]. Although marine transportation industries continue to grow, there is a concomitant increase in the number of marine transportation accidents. The expansion of port facilities, ship operations, and the properties of HAZMAT that the ships intend to transport typically contributed an adverse impact onto the occurrence of accidents. By transporting HAZMAT in bulk quantity via specially designed ships, it is highly important to understand the risks and hazards associated with the newly marine transportation operations especially during the loading, en-route, and unloading. Therefore, a comprehensive assessment is required for operational safety in the marine transportation of HAZMAT. Therefore, the underlying question that would like to be addressed in this study is whether the respective contributing factors are sufficiently identified or not.

Various studies have been done in order to avoid the re-occurrence of similar accident cases involving the marine 
transportation of HAZMAT. Many accidents were reported like sinking, grounding, fire, explosion, and collision-contact [2,3]. Although the eventuality of accidents involving HAZMAT transport is relatively low, several examples of past accidents worldwide, such as the MSC Rosa M in 1997, Ever Decent in 1999, Napoli in 2007, and Princess of the Stars in 2009demonstrated that there exists the possibility of these accidents to recur[4]. It is also important to note that the most significant accident results in terms of life lost, injuries, and environmental pollutions are often not specified, and the debates are still much on the various contributing factors in the occurrences of these accidents. Therefore, the marine transportation industries need to emphasize inherently safer daily operation for HAZMAT transportation. According to Mawson [5], safe transportation of HAZMAT is one of the most serious challenges that require huge commitments from various parties such as the authorities, NGOs, and industries. These commitments can be achieved through adequate technologies and proper control measures to improve marine safety, mainly in the context of HAZMAT operation.

Thus, this study provides an overview of the factors that contributed to HAZMAT-related accidents in marine transportation. In this study, the focus mainly evolves in identifying and prioritizing the factors that should be assessed in the early stage of safety assessment in the marine transportation operation. Section 2 of this study presents the detail description of the methodology, which is based on an actual survey. Section 3 illustrates briefly the results and discussion, and Section 4 describes the conclusion.

\subsection{METHODOLOGY}

This section presents the methodology that has been applied in this study (Fig.1). Basically, the methodology consists of two main steps: (1) identification of the contributing factors based on literature review and (2) administration of the survey.

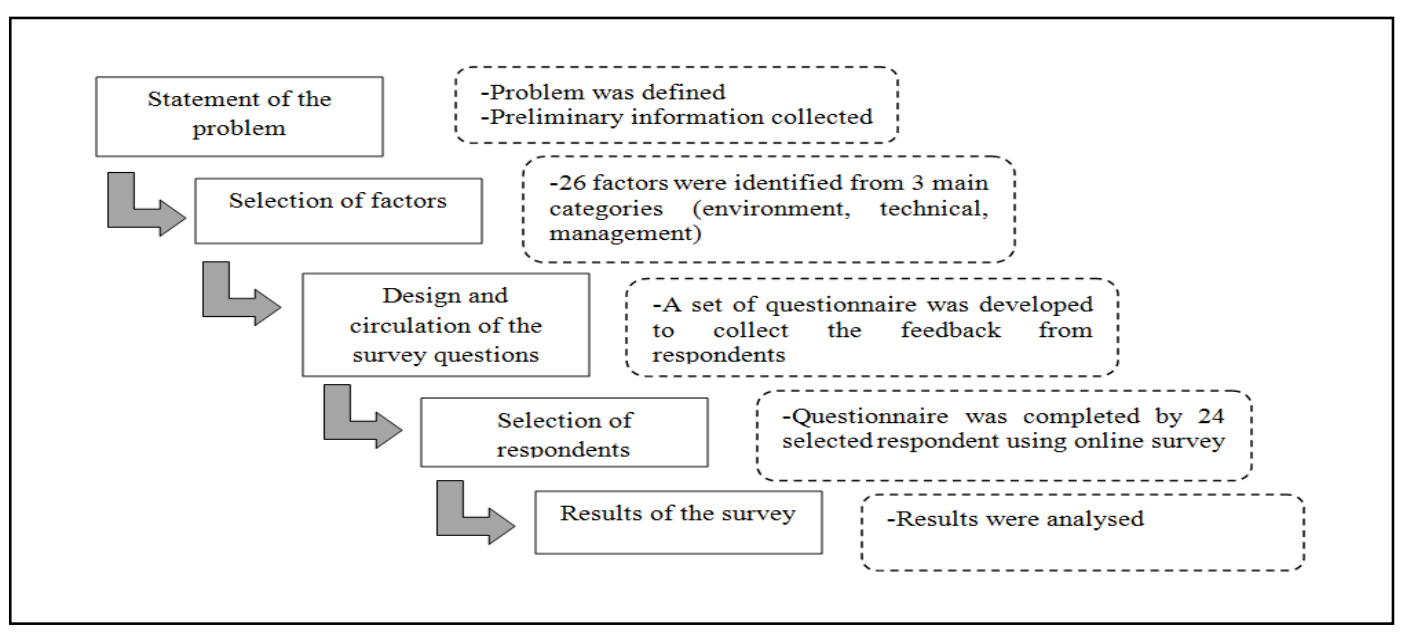

Figure 1. Diagram of methodology

An extended review was performed among some of the most well-known and reliable accident databases. For instance, marine accident databases such as the Marine Hazard Incident Database System (MHIDAS) and the Marine Accident Investigation Branch (MAIB) of the UK Department of Transport were referred extensively. Moreover, several documents including the reports of marine accidents produced by the Swedish authority (2002-2008) as well as a statistical update of marine accidents by the Malaysian Marine Department (2008-2011) were used as the source of information on accident-related data.These allow the identification of the factors that are important determinants of marine transportation accidents. Basically, the necessary information regarding the number and characteristics of accidents, as well as the extent of their consequences, mainly in terms of human fatalities and injuries were also taken into account for the present study. From the safety point of view, it is one of the crucial steps because of the characteristics of marine transportation operation. The existence of unforeseen hazards in the operation has the potential to constitute the expansion of different causes of accidents, which are difficult to determine in the early stage of safety assessment. Each cause may be subjected to various uncertainties due to data limitations. All the related information on the contributing factors of marine transportation accident is summarized in Fig.2. 


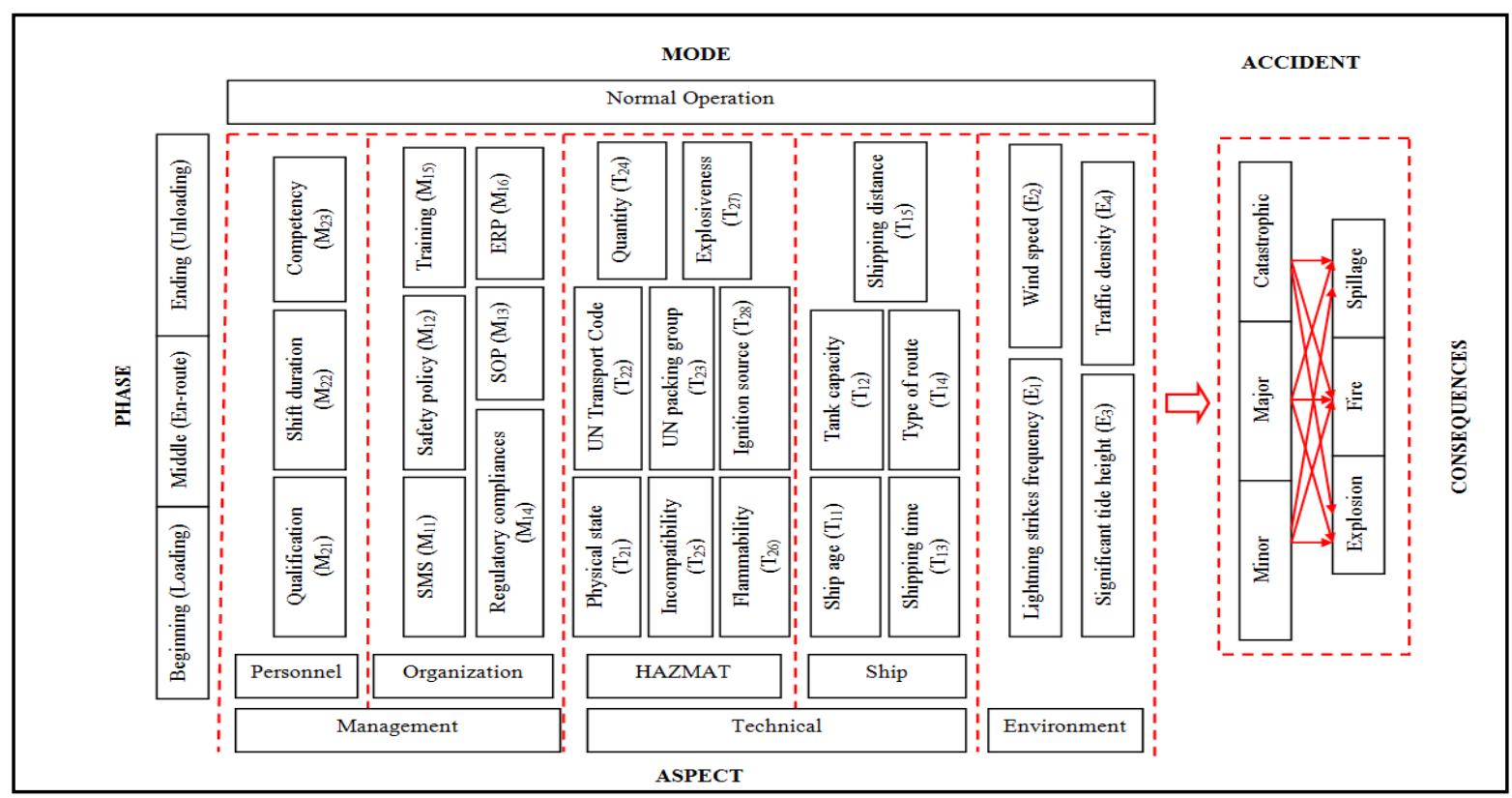

Figure 2. Causal factors of marine transportation accident

Fig.2 shows the categorization of 26 factors identified from the previous step that belong to any of the three main categories: environment, technical, and management. As designed initially, a set of questionnaire was constructed based on the list of questions as follows:

\section{Environmental Category:}

$\mathrm{E}_{1}$ Are there prone route(s) for lightning strikes?

$\mathrm{E}_{2}$ Is wind speed measured?

$\mathrm{E}_{3} \mathrm{Is}$ the sea state (significant tide height) recorded?

$\mathrm{E}_{4} \mathrm{Has}$ the traffic density of shipping route been recorded?

\section{Technical Category:}

$\mathrm{T}_{11}$ Is the ship in good conditions?

$\mathrm{T}_{12} \mathrm{Is}$ the tank capacity of the ship properly checked?

$\mathrm{T}_{13}$ Are shipping times been recorded during the operation of HAZMAT transport?

$\mathrm{T}_{14}$ Are the types of shipping route determined?

$\mathrm{T}_{15}$ Has the shipping distance been determined?

$\mathrm{T}_{21}$ Are the properties of materials being observed?

$\mathrm{T}_{22}$ Have the UN transportation code requirements been followed?

$\mathrm{T}_{23}$ Have the UN packing group requirements been followed?

$\mathrm{T}_{24}$ Is gross weight of materials properly recorded?

$\mathrm{T}_{25}$ Is the material (incompatibility) securely controlled?

$\mathrm{T}_{26}$ Is the material (flammability) securely controlled?

$\mathrm{T}_{27}$ Is the material (explosiveness) securely controlled?

$\mathrm{T}_{28}$ Are the ignition sources existed?

\section{Management Category:}

$\mathrm{M}_{11}$ Have efforts been taken to establish safety management system (SMS)?

$\mathrm{M}_{12} \mathrm{Is}$ the appropriate safety policy being used?

$\mathrm{M}_{13}$ Have efforts been taken to implement the standard operating procedure (SOP) for HAZMAT transport?

$\mathrm{M}_{14} \mathrm{Has}$ commitment been taken to comply with the regulations related to HAZMAT transport?

$\mathrm{M}_{15} \mathrm{Has}$ an adequate and comprehensive training been provided to the ship crews?

$\mathrm{M}_{16}$ Are there sufficient monitoring and updating on emergency response preparedness during HAZMAT transport operation? 
$\mathrm{M}_{21}$ Are all ship crews involved in operations qualified andunderstood the hazards and risks of HAZMAT transport?

$\mathrm{M}_{22} \mathrm{Have}$ there been poor/disturbed health conditions (tiredness, sleepy, fatigue) among ship crews due to shift rotation (day/night shift)?

$\mathrm{M}_{23}$ Have there been decision errors committed by ship crews due to lack of competency?

Each factor listed above was structured based on the tendency of marine transportation accident that was rated using a scale as follows:

- $\quad$ "1" : The least significant factor

- $\quad$ "2" : Partially significant factor

- $\quad$ "3" : Significant factor

- $\quad$ "4" : More significant factor

- $\quad$ "5" : The most significant factor

For the next step, a pilot study was conducted to determine the reliability value of Cronbach's alpha of the questionnaire. A total of 10 marine industrial respondents were chosen for the pretesting of the questionnaire [6]. Then, an actual survey was distributed among 50 selected respondents who were involved directly or indirectly in the marine transportation field in Malaysia through an online survey. These responses were used to provide a clearer picture of the actual situations and difficulties faced particularly in the operation of marine transportation of HAZMAT. In other words, the average value and importance index were calculated to indicate the extent to which the respondents believed that these factors contributed to the occurrence of marine transportation accidents. These analyses were also applied to determine whether differences in score exist across the feedback of the respondents on the priority applied for ranking the factors. A high score on the importance index indicates that the respondents perceived high contributions of the factor in causing marine accidents, and a low score vice versa. In order to rank each factor according to their appropriate ranking point, the methods of calculating the average value and importance index are represented by using Equation 1 and 2 as follows:

$$
\text { Average Value }=\sum_{\underline{i=1}}^{n}\left(a_{i} s_{i}\right)
$$

where $a_{1}, a_{2}, a_{3}, a_{4}$, and $a_{5}$ indicate the number of responses regarding the scoring scale of $1,2,3,4$, and 5 respectively. Meanwhile, the importance index is written as:

$$
\text { Importance Index }=\underline{\text { Average value }} \times 100 \%
$$

where $\mathrm{Z}$ indicates the highest value on a scoring scale.

In order to be more intuitive, this study applied an approach called a Green-Yellow-Red list to indicate the level of importance of each factor. The range of score that was formed initially for the purposes of the calculation is presented in Table 1.

Table 1. Range of score

\begin{tabular}{cc}
\hline Range of Score (\%) & Level of Importance \\
\hline$<50$ & Low \\
$50-74$ & Medium \\
$75-100$ & High \\
\hline
\end{tabular}




\subsection{RESULTS AND DISCUSSION}

Based on the pilot study, the reliability of the questionnaire was analyzed using the Statistical Package Science (SPSS) 22.0 software. The result indicates that the level of reliability of the data was excellent, with the value of Cronbach's alpha of 0.920 . According to the published guideline [7], a value more than 0.8 is considered highly acceptable, and it should not be less than 0.7 , as it is the standard criteria for the reproducibility of the questionnaire.

For the results of an actual survey, data screening was initially performed. After data cleaning and the removal of incomplete data, only 24 out of 50 surveys were included in the final data analysis, and these gave the overall response rate of only $48 \%$. Meanwhile, the results of average value and importance index calculation are summarized in Table 2. According to these results, eight out of 26 factors were within high level, 14 factors were evaluated as medium, and the other four factors including lightning strikes frequency $\left(E_{1}\right)$, shipping time $\left(E_{2}\right)$, wind speed $\left(T_{13}\right)$, and shipping distance $\left(T_{15}\right)$ were indicated as low according to the calculation conducted.

In summary, the operational safety level of marine transportation of HAZMAT is primarily influenced by the traffic density, classification of the UN Transport Code and UN packing group, safety management system (SMS), standard operating procedure (SOP), training, shift duration, and also competency. Using the results below, when these data are available, the appropriate ranking point for each factor is able to improve the existing safety assessment in HAZMAT operation. With these approaches, the risk of HAZMAT marine transport during the activities such as preparation of HAZMAT for transportation, packaging, and loading the ship can be managed directly through effective control measures and preparing action plans in mitigating the consequences of possible accidents. Indirectly, the related hazards and risks can be predicted to prevent any unintended situation that may lead to an accident.

Table 2. Level of importance of factor

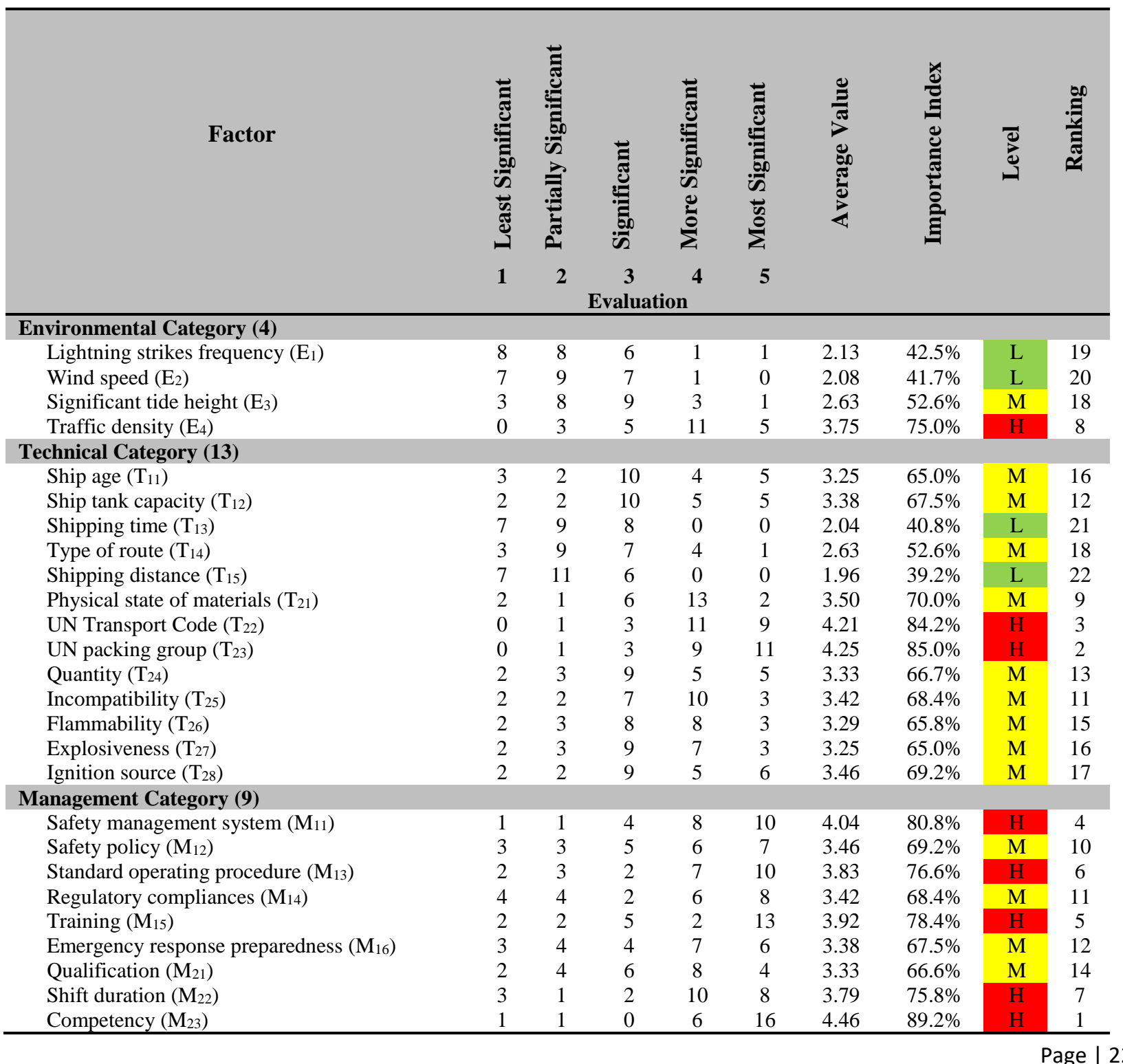




\subsection{CONCLUSION}

In this study, the ranking point for each factor on HAZMAT-related accidents in marine transportation is presented. It gives a new and imperative output that the academics and shipping industries have barely touched on. As for a new contribution, the level of importance was calculated for each factor that belongs to any of the three main categories: environment, technical, and management. In other words, it can assist shipping industries to focus on the loopholes of existing safety assessment specifically for HAZMAT transportation, in order to provide an initial screening as well as identifying potential hazards and risks in the early phase of marine transport operation. Therefore, a comprehensive safety assessment in marine transportation of HAZMAT can be performed.

\section{References}

[1] United Nations Conference on Trade and Development. 2011. 'Review of Maritime Transport'. UCTAD, Geneva.

[2] Kristiansen, S. 2005. 'Maritime Transportation Safety Management and Risk Analysis'. Burlington: Biddles.

[3] Wang, J., Pillay, A., Kwon, Y.S., Wall, A.D., Loughran, C.G. 2005. An Analysis of Fishing Vessel Accidents. Accident Analysis and Prevention. 37 (6): 1019-1024.

[4] Mamaca, E., Girin, M., Le Floch, S., Le Zir, R. 2009. 'Review of Chemical Spills at Sea and Lessons Learnt'. A Technical Append for the Interspill 2009 Conference White Paper.

[5] Mawson, J.P. 2003. 'Issues Arising from the Development in Design and Operation of Container Ships. The Impact on the Safe Carriage of Goods and Crew'. MA Dissertation. University of Greenwich, Greenwich Maritime Institute, Greenwich, UK.

[6] Baker, T. L. 1994. Doing Social Research (2 ${ }^{\text {nd }}$ Ed.). New York: McGraw-Hill Inc.

[7] George, D., Mallery, P. (2003). SPSS for Windows Step By Step: A Simple Guide and Reference. 11.0 update. $4^{\text {th }}$ ed. Boston: Allyn \& Bacon. 